\title{
Polarization change due to fast winds from accretion disks
}

\author{
Andrei M. Beloborodov ${ }^{1,2}$,
}

\begin{abstract}
A fraction of the radiation produced by an accretion disk may be Thomson scattered by a wind flowing away from the disk. Employing a simple plane-parallel model of the wind, we calculate the polarization of the scattered radiation and find that its sign depends on the wind velocity, $\beta=v / c$. In the case, $0.12<\beta<0.78$, the polarization is parallel to the disk normal, i.e., it is orthogonal to the standard Chandrasekhar's polarization expected from accretion disks. The velocity of an $e^{ \pm}$wind is likely to saturate near the equilibrium value $\beta_{*} \sim 0.5$ for which the accelerating radiation pressure is balanced by the Compton drag. Then the change of polarization by the wind is most pronounced. This may help to reconcile the standard accretion disk model with the optical polarimetric observations of non-blazar AGNs.
\end{abstract}

Subject headings: accretion, accretion disks - polarization - radiative transfer - scattering

\section{Introduction}

The standard model of a black hole accretion disk predicts the polarization of the emerging radiation. The radiation gets polarized due to electron scattering that strongly dominates the absorption opacity in luminous accretion disks. Polarization of light emerging from an electron plane-parallel atmosphere was calculated by Chandrasekhar (1960) and Sobolev (1963). According to their results, the polarization should be parallel to the disk surface with a maximum of $11.7 \%$ when the disk is viewed edge-on. This simple model, however, disagrees with observations: the optical polarization in non-blazar AGNs is typically $\sim 1 \%$ or less, and it tends to be parallel to the radio jet that is presumably perpendicular to the disk (Stockman, Moore, \& Angel 1984; Antonucci 1992). The problem of accretion-disk polarization has been discussed by a number of authors (e.g. Gnedin \& Silant'ev 1978; Loskutov \& Sobolev 1981; Sunyaev \& Titarchuk 1985; Phillips \& Mészáros 1986; Coleman \& Shields 1990; Laor, Netzer, \& Piran 1990; Matt, Fabian, \& Ross 1993; Kartje 1995; Agol \& Blaes 1996). In this Letter, we suggest that the original polarization of the disk can be changed due to Thomson scattering in a mildly relativistic wind.

\footnotetext{
${ }^{1}$ Stockholm Observatory, S-133 36, Saltsjöbaden, Sweden

${ }^{2}$ Astro-Space Center of Lebedev Physical Institute, Profsoyuznaya 84/32, Moscow 117810, Russia
} 
Gas outflows are observed in non-blazar AGNs, and evidence for mildly relativistic bulk velocities has been reported (Leighly et al. 1997). The outflows are seen on scales far exceeding the scale of the central source $\sim 10^{14} \mathrm{~cm}$, and the gas dynamics in the central region is poorly understood. It is quite probable that there is a wind from the innermost region of an accretion disk where the bulk of the observed radiation originates. The wind may form due to a gas outflow from a corona of the disk. It may also form due to an outflow of electron-positron pairs produced by the gamma-rays from the disk. In the latter case, the created pairs cool efficiently by inverse Compton scattering down to the Compton temperature $k T_{C} \sim 1-10 \mathrm{keV}$, and the cool light $e^{ \pm}$plasma is then easily pushed away by the radiation pressure.

To investigate the effects that a wind could introduce in the pattern of polarization, we consider the simplest plane-parallel model in which the disk is replaced by a homogeneously emitting plane and the wind is assumed to be a cold vertical outflow from the plane (by "cold" we mean that the thermal motions of particles in the outflow are slow compared to the bulk motion). Thomson scattering of frequency-integrated polarized radiation is governed by the transfer equations written down in $\S 2$. The equations account for the relativistic aberration of light in the wind rest frame, which is crucial for the polarization due to scattering. In $\S 3$ and $\S 4$ we discuss in turn the optically thin and optically thick winds. The results are summarized in $\S 5$.

\section{Basic equations}

In a plane-parallel slab, the polarized radiation is represented by the frequency-integrated intensities $I_{l}, I_{r}$, where the index $l$ refers to the radiation polarized in the meridional plane (defined by the normal and the ray), and index $r$ refers to the polarization perpendicular to this plane. The radiation is axisymmetric with respect to the normal, with the intensity being a function of $\mu-$ the cosine of the angle between the ray and the normal. Stationary Thomson transfer in a cold medium moving in the vertical direction with a velocity $\beta=v / c$ is described by the equations,

$$
\mu \frac{\partial I}{\partial \tau}=(1-\beta \mu)(I-S), \quad \mu \frac{\partial Q}{\partial \tau}=(1-\beta \mu)(Q-R) .
$$

Here, $\tau$ is the scattering optical depth that the medium would have at rest, $I=I_{r}+I_{l}$, and $Q=I_{r}-I_{l} . S$ and $R$ are source functions representing the scattered radiation.

A simple way to derive the source functions is to transform the radiation field to the wind rest frame where Thomson scattering is coherent. Polarization is invariant with respect to Lorentz boosts along the normal, and the frequency-integrated intensities $I$ and $Q$ transform as (see, e.g., Rybicki \& Lightman 1979)

$$
\begin{gathered}
I^{c}\left(\mu_{c}\right)=D^{4} I(\mu), \quad Q^{c}\left(\mu_{c}\right)=D^{4} Q(\mu), \\
\mu_{c}=\frac{\mu-\beta}{1-\beta \mu}
\end{gathered}
$$


where the index $c$ stands for the comoving frame, $D=\gamma(1-\beta \mu)$ is the Doppler factor, $\gamma$ is the Lorentz factor. When viewed from the comoving frame, the scattered radiation is represented by the source functions, $S^{c}\left(\mu_{c}\right)$ and $R^{c}\left(\mu_{c}\right)$, given by Sobolev (1963). Transforming the scattered radiation back to the laboratory frame, $S(\mu)=D^{-4} S^{c}\left(\mu_{c}\right)$ and $R(\mu)=D^{-4} R^{c}\left(\mu_{c}\right)$, we get

$$
\begin{gathered}
S(\mu)=\frac{1}{D^{4}}\left[I_{0}^{c}+\frac{3}{8}\left(3 \mu_{c}^{2}-1\right)\left(I_{2}^{c}-\frac{1}{3} I_{0}^{c}+Q_{0}^{c}-Q_{2}^{c}\right)\right], \\
R(\mu)=\frac{9\left(1-\mu_{c}^{2}\right)}{8 D^{4}}\left(I_{2}^{c}-\frac{1}{3} I_{0}^{c}+Q_{0}^{c}-Q_{2}^{c}\right),
\end{gathered}
$$

where $I_{m}^{c}, Q_{m}^{c}(m=0,1,2)$ are the moments of the radiation field in the comoving frame,

$$
I_{m}^{c}=\frac{1}{2} \int_{-1}^{1} I^{c}\left(\mu_{c}\right) \mu_{c}^{m} \mathrm{~d} \mu_{c}, \quad Q_{m}^{c}=\frac{1}{2} \int_{-1}^{1} Q^{c}\left(\mu_{c}\right) \mu_{c}^{m} \mathrm{~d} \mu_{c}
$$

$I_{m}^{c}$ are related to the corresponding moments in the lab frame, $I_{m}$, by

$$
\begin{gathered}
I_{0}^{c}=\gamma^{2}\left[I_{0}-2 \beta I_{1}+\beta^{2} I_{2}\right], \quad I_{2}^{c}=\gamma^{2}\left[\beta^{2} I_{0}-2 \beta I_{1}+I_{2}\right], \\
I_{1}^{c}=\gamma^{2}\left[-\beta\left(I_{0}+I_{2}\right)+\left(1+\beta^{2}\right) I_{1}\right] .
\end{gathered}
$$

$Q_{m}$ transform in exactly the same way. Note that $Q_{0}^{c}-Q_{2}^{c}=Q_{0}-Q_{2}$.

In a $2 \mathrm{D}$ approach, an initially vertical wind becomes quasi-radial at some height and its opacity falls off. We consider the simplified 1D problem in which the wind is replaced by a slab of vertically outflowing plasma of a given optical depth $\tau_{0}$. At the outer boundary, $\tau=0$, we assume free escape of the radiation. At the inner boundary, $\tau=\tau_{0}$, we assume the existence of a source of limbdarkened positively polarized radiation (Chandrasekhar 1960 ) for which $I_{1} \approx 0.581 I_{0}, I_{2} \approx 0.414 I_{0}$.

\section{Optically thin wind}

In the case of a small optical depth, $\tau_{0}$, the optically thin approximation holds at angles satisfying the condition, $\tau_{\mu}=\tau_{0}(1-\beta \mu) / \mu<1$, and equations (1) yield the scattered radiation emerging from the slab as

$$
I_{s c} \approx \tau_{\mu} S(\mu), \quad Q_{s c} \approx \tau_{\mu} R(\mu)
$$

The polarization degree of the scattered radiation equals $p_{s c}(\mu)=Q_{s c} / I_{s c}=R / S$. From equations (2) and (3) we get

$$
p_{s c}(\mu)=\frac{3\left(1-\mu_{c}^{2}\right) \eta}{(8 / 3)+\left(3 \mu_{c}^{2}-1\right) \eta}
$$

where we have introduced the parameter

$$
\eta=\frac{I_{2}^{c}-\left(I_{0}^{c} / 3\right)+Q_{0}-Q_{2}}{I_{0}^{c}} \approx \frac{I_{2}^{c}}{I_{0}^{c}}-\frac{1}{3} .
$$


The contribution to $\eta$ from $Q_{0}$ and $Q_{2}$ is $\sim 30$ times smaller than that from $I_{2}^{c}-I_{0}^{c} / 3$, and can be neglected. The parameter $-1 / 3<\eta<2 / 3$ shows to what extent the radiation field is stretched along the normal in the rest frame of the wind, which is of crucial importance for the polarization of the scattered radiation as $p_{s c}(\mu)$ has the same sign as $\eta$ at all $\mu$. In Figure 1 we show the dependence of $\eta$ on the wind velocity $\beta: \eta$ changes sign at $\beta_{1} \approx 0.12$ and $\beta_{2} \approx 0.78$. It follows that the scattered radiation remains positively polarized if the wind is slow, $\beta<\beta_{1}$. The change in sign of the polarization happens if the wind has a velocity in the range, $\beta_{1}<\beta<\beta_{2}$.

In the case the outflow is comprised of a light $e^{ \pm}$plasma, the time-scale to achieve Compton equilibrium with the radiation field is small and the wind velocity adjusts so that the radiation pressure is balanced by the Compton drag (see, e.g., Phinney 1982). It means that, in the comoving frame, the net radiation flux vanishes, i.e., $4 \pi I_{1}^{c}=0$, and the equation for the equilibrium velocity, $\beta_{*}$, reads

$$
\beta_{*}\left(I_{0}+I_{2}\right)=\left(1+\beta_{*}^{2}\right) I_{1},
$$

which yields $\beta_{*} \approx 0.52$ for the limb-darkened radiation from the disk. One can check that $\eta(\beta)$ has a minimum at $\beta=\beta_{*}$. It follows that a wind produces the strongest negative polarization if it is in Compton equilibrium with the radiation field. This fact is illustrated by the diagram embedded in Figure 1 that shows the angular distribution of the disk radiation in the rest frame of the wind. A special feature of the aberration at mildly relativistic $\beta$ is that the radiation field is effectively "compressed" in the vertical direction. It is this compression that results in parallel polarization of the scattered radiation, and the compression is naturally strongest at $\beta=\beta_{*}$ for which the net flux in the comoving frame vanishes.

Whatever velocity of the wind, $\beta$, the scattered radiation has an extremum of polarization at $\mu=\beta$. It corresponds to $\mu_{c}=0$, i.e., to the case the ray is perpendicular to the normal in the comoving frame, and we denote the extremum as $p_{s c}^{\perp}$. In Figure $1, p_{s c}^{\perp}$ is plotted against $\beta$. For a mildly relativistic wind, $p_{s c}^{\perp} \approx \eta$, e.g., $p_{s c}^{\perp}=-19 \%$ for $\beta=\beta_{*}$. In the ultrarelativistic limit, the aberrated radiation gets concentrated in "head-on" direction and $p_{s c}^{\perp} \rightarrow 100 \%$, as discussed by Begelman \& Sikora (1987).

An observer viewing a disk with an optically thin outflow will see the sum of unscattered and once scattered radiation. In Figure 2 we plot the observed polarization as a function of the disk inclination, $\mu$, for the case $\tau_{0}=0.1$ and $\beta=\beta_{*}=0.52$. The optical depth is small and the scattering in the wind weakly affects the intensity of the observed radiation. One can see, however, that the wind changes the pattern of polarization. This happens because the polarization extremum of the scattered radiation is boosted into angles $\mu \sim 0.5$, where the original disk polarization is small. Note that the calculated slab model fails at small $\mu$ as $\tau_{\mu}$ increases $\propto \mu^{-1}$ to $\tau_{\mu}=1$ where the single scattering approximation is not applicable. In a real wind, the scattering region has typical height comparable to its radius, and a detailed 2D model is needed to get exact pattern of polarization. To illustrate importance of geometry, take a cylinder of height $h$ and radius $r=\sqrt{3} h$ instead of slab. Then, at $\mu>0.5, \tau_{\mu}$ changes to $\tau_{\mu}=\sqrt{3} \tau_{0}(1-\beta \mu)\left(1-\mu^{2}\right)^{-1 / 2}$. Combined with the slab source functions, $S$ and $R$, this yields a rough estimate for the resulting polarization shown by the 
dotted line in Figure 2.

\section{Optically thick wind}

An optically thick $e^{ \pm}$outflow may emerge in the case a fraction of the disk luminosity is emitted above $511 \mathrm{keV}$, as hard photons are absorbed by softer X-rays to produce $e^{ \pm}$pairs (the nonlinear transfer of gamma-rays above a luminous disk is discussed in Beloborodov 1998). Compton equilibrium is established in each layer of the outflow, and the bulk velocity is determined by the radiation field itself through the equilibrium condition (6). This means that the velocity should be calculated self-consistently in the transfer problem. Comparing the Compton equilibrium transfer

to the classical transfer problem in an electron medium at rest, we note that in addition to a constant net flux, $4 \pi I_{1}(\tau)=$ const, we have $I_{2}(\tau)=$ const (one can check this combining the first moment of the transfer equation [1] with the equilibrium condition [6]). $4 \pi I_{2} / c$ equals the radiation pressure in the vertical direction, and its constancy reflects the condition that the net radiative force acting on $e^{ \pm}$vanishes in the limit $\beta \rightarrow \beta_{*}$. The equilibrium velocity varies with height, and pairs keep $\beta \approx \beta_{*}$ as $\beta_{*}$ is a strong attractor in phase space.

We have simulated this transfer problem numerically. We take as initial conditions that the disk radiation is propagating freely in the slab, then "switch on" the scattering by the flowing pairs at $t=0$, and follow the evolution of the system until a stationary solution is established. In the calculations, we use a grid which is homogeneous in the $\cos ^{-1} \mu$ and $\tau$ directions. The number of grid points is $N_{\tau} \times N_{\mu}=200 \times 300$. The stationary solution, $I(\tau, \mu)$ and $Q(\tau, \mu)$, that we are looking for depends only on the parameter $\tau_{0}$ and does not depend on the $e^{ \pm}$density profile, $n(\tau)$. Therefore, we choose the simplest profile, $n(\tau)=$ const. The chosen time step equals $\Delta t=0.5 \tau_{0} / n \sigma_{\mathrm{T}} c N_{\tau}$.

The stationary solution for $\tau_{0}=3$ is shown in Figures 3 and 4 . Figure 3 displays the wind velocity. In the lowest layers of the slab, the equilibrium velocity is less than $0.5 c$ since besides the disk radiation there is some flux in the opposite direction due to radiation being back scattered by the outflow. In higher layers, the radiation gets collimated by the scattering on the moving pairs, and the equilibrium velocity increases to $\sim 0.7 c$. Figure 4 shows the radiation emerging from the slab: it is significantly anisotropic, and it is negatively polarized with an extremum of $p \approx-14 \%$ at $\mu \approx 0.43$.

\section{Conclusions}

We conclude that due to Thomson scattering in a wind, the disk radiation originally polarized perpendicular to the normal can acquire parallel polarization. This effect arises due to relativistic aberration of light in the wind rest frame and takes place when the wind velocity is in the range $0.12<\beta<0.78$. Being characteristic for mildly relativistic winds, the production of parallel polarization does not occur in the previously discussed scattering by highly relativistic jets (Begelman 
\& Sikora 1987) which produce strongly beamed radiation with polarization perpendicular to the jet.

An observer viewing a disk through an optically thin wind will see the sum of the radiation from the disk and the radiation scattered in the wind. If the original disk radiation has the perpendicular Chandrasekhar-Sobolev polarization then the scattering in the wind tends to diminish it. The strongest effect is produced by a wind with velocity $\beta=\beta_{*} \sim 0.5$, where $\beta_{*}$ corresponds to a Compton equilibrium of the outflow with the disk radiation field. Such an equilibrium is likely to be established in a wind comprised of an $e^{ \pm}$plasma. We find in this case that a modest optical depth of the wind, $\sim 0.1$, is enough to change the pattern of the disk polarization: The polarization degree is reduced and there appears a range of inclination angles with negative (parallel) polarization. This change may naturally explain the optical polarization observed in non-blazar AGNs.

We have also found that parallel polarization is produced in an optically thick $e^{ \pm}$outflow which might form above a black hole accretion disk. The outflow is semi-relativistic, and the emerging radiation is markedly beamed along the disk axis. The bulk of the disk radiation gets scattered, its original polarization is lost, and the emerging radiation acquires parallel polarization with an extremum of about $-14 \%$ at an inclination angle of $\sim \pi / 3$. Note that an optically thick $e^{ \pm}$wind also produces an annihilation feature above $511 \mathrm{keV}$, which has a blueshift up to $\sim 2$, albeit smeared out by the velocity gradient in the wind.

Our general conclusion is that a wind produces parallel polarization when it is near Compton equilibrium with the radiation field. This conclusion is derived for vertical winds in slab geometry and it remains to be investigated how it is affected by deviations from this simple model. In particular, a magnetic field may affect the wind direction, especially if magnetic pressure dominates the radiation pressure, and additional relativistic effects are important near Kerr black holes. A detailed 2D model should include a radial dependence of the disk emission and the wind density.

I thank C.-I. Björnsson, J. Poutanen, and R. Svensson for many useful remarks, and the referee for helpful comments. I acknowledge support from the Swedish Natural Science Research Council and RFFI grant 97-02-16975. 


\section{References}

Agol, E., \& Blaes, O. 1996, MNRAS, 282, 965

Antonucci, R.R.J. 1992, in Testing the AGN paradigm, ed. S. Holt, S. Neff, C.M. Urry (New York), 486

Begelman, M.C., \& Sikora, M. 1987, ApJ, 322, 650

Beloborodov, A.M. 1998, MNRAS, submitted

Chandrasekhar, S. 1960, Radiative Transfer (New York: Dover)

Coleman, H.H., \& Shields, G.A. 1990, ApJ, 363, 415

Gnedin, Y.N., \& Silant'ev, N.A. 1978, SvA, 22, 325

Kartje, J.F. 1995, ApJ, 452, 565

Laor, A., Netzer, H., \& Piran, T. 1990, MNRAS, 242, 560

Leighly, K.M., Mushotzky, R.F., Nandra, K., \& Forster, K. 1997, ApJ, 489, L25

Loskutov, V.M., \& Sobolev, V.V. 1981, Astrofisika, 17, 535

Matt, G., Fabian, A.C., \& Ross, R.R. 1993, MNRAS, 264, 839

Phillips, K.C., \& Mészáros, P. 1986, ApJ, 310, 284

Phinney, S. 1982, MNRAS, 198, 1109

Rybicki G.B., \& Lightman A.P., 1979, Radiative Processes in Astrophysics (New York: Wiley)

Sobolev, V.V. 1963, A Treatise on Radiative Transfer (Princeton: Van Nostrand)

Stockman, H.S., Moore, R.L., \& Angel, J.R.P. 1984, ApJ, 279, 485

Sunyaev, R.A., \& Titarchuk, L.G. 1985, A\&A, 143, 374 


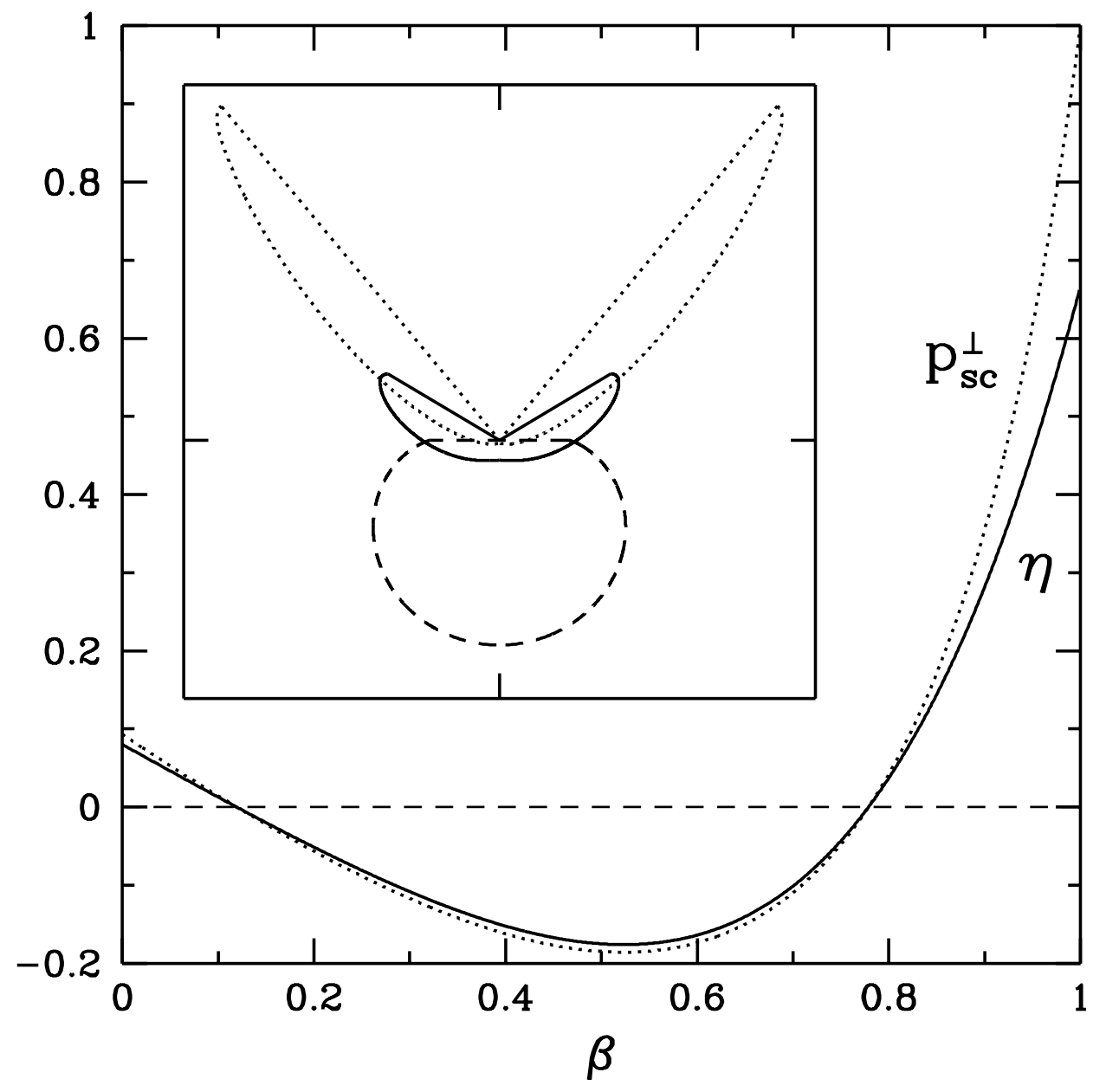

Fig. 1.- Dependence of parameter $\eta$ on the wind velocity, $\beta$, (solid curve). The disk is modeled as a plane-parallel electron atmosphere emitting limb-darkened radiation (Chandrasekhar 1960). The dotted curve displays $p_{s c}^{\perp}$, the degree of polarization of the radiation scattered at right angle to the normal in the comoving frame. The embedded diagram shows the angular distribution of the aberrated radiation seen by an electron in the wind, $I^{c}(\mu)$. Dashed, solid, and dotted lines correspond to $\beta=0, \beta=\beta_{*}=0.52$, and $\beta=\beta_{2}=0.78$ respectively. 


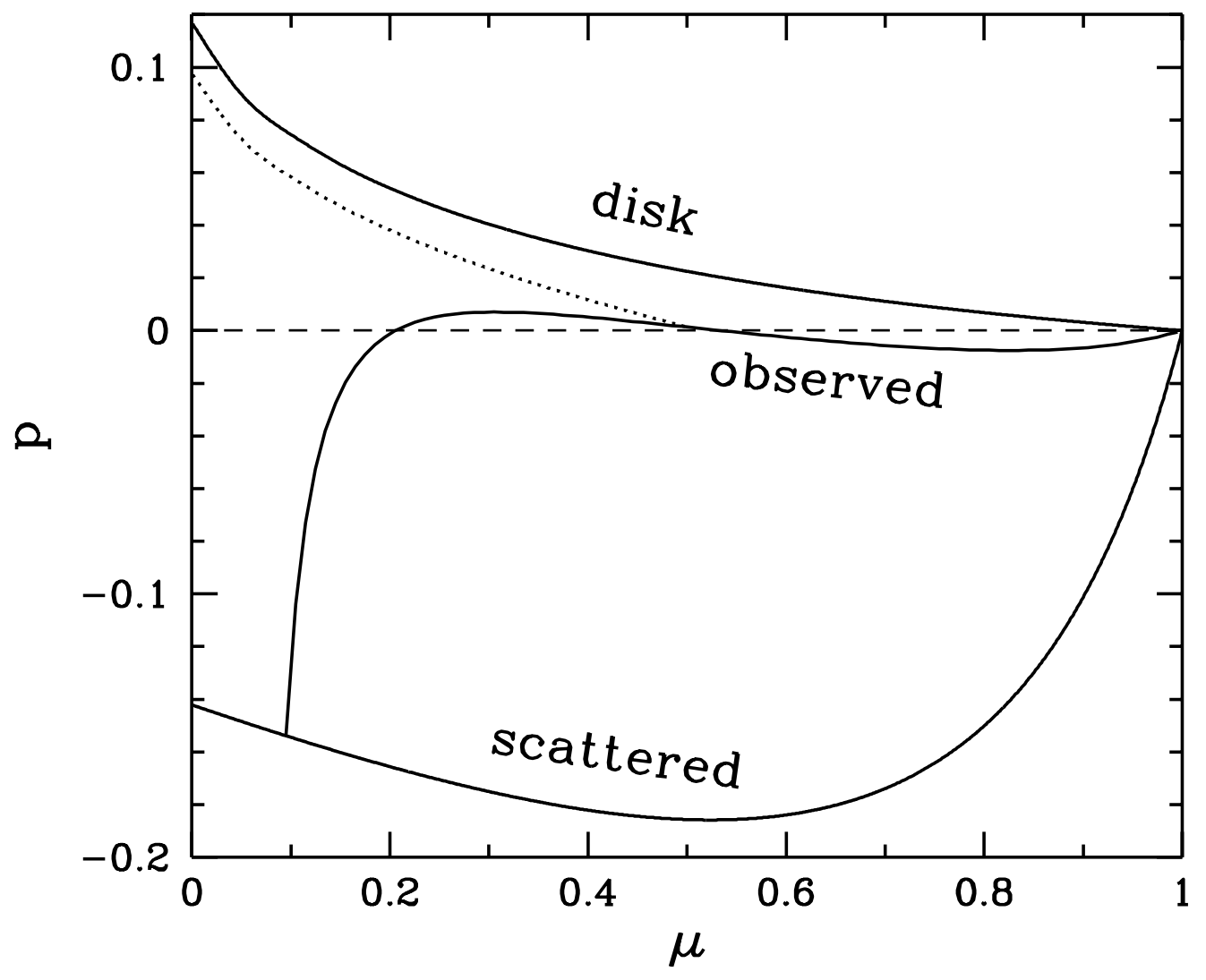

Fig. 2.- Angular dependence of the polarization from an accretion disk with a wind of optical depth $\tau_{0}=0.1$ and velocity $\beta_{*}=0.52$. The scattered radiation is calculated in the single scattering approximation which fails for the slab model (solid line) when the "observed" curve approaches the "scattered" one at $\mu \sim 0.1$. The estimate for the cylinder model is shown by the dotted line. 


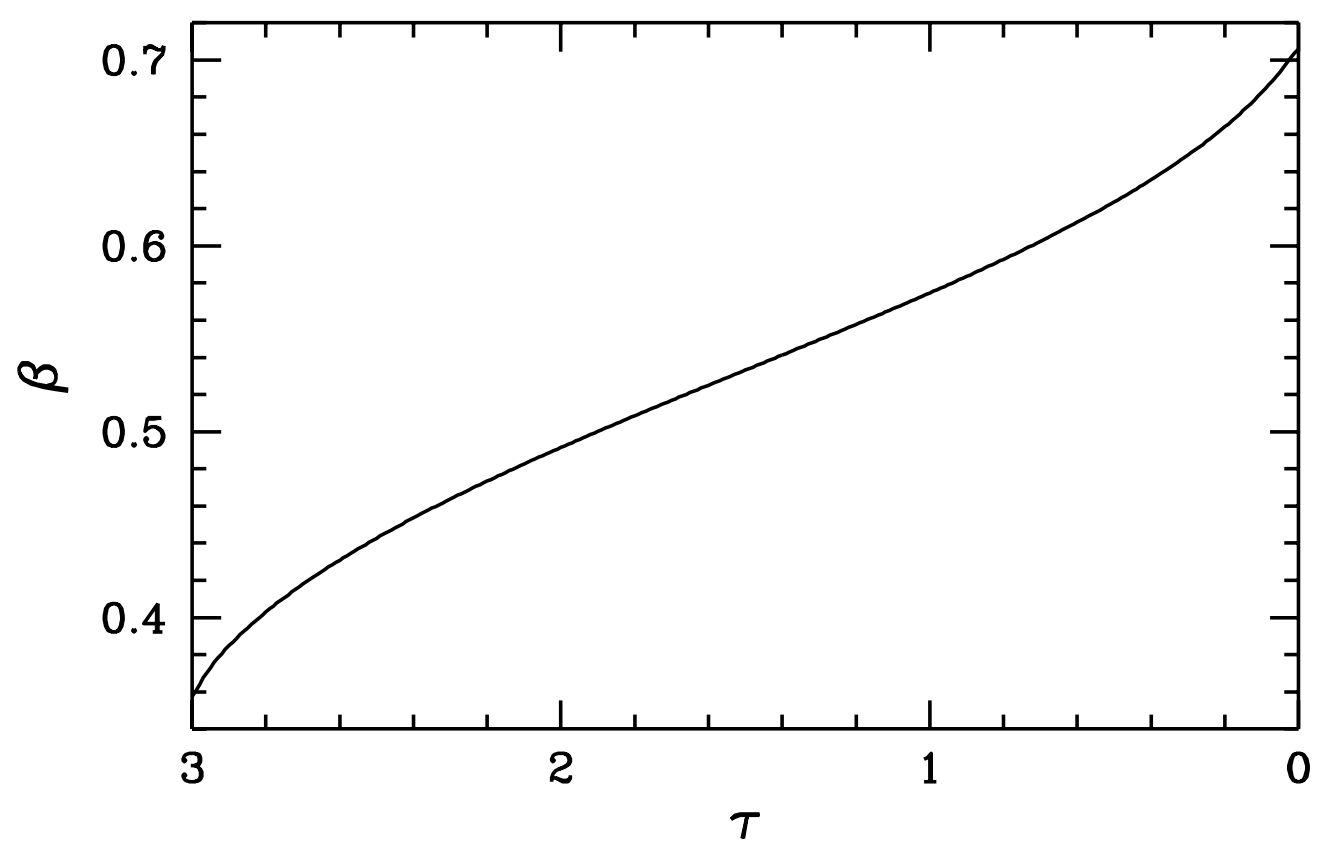

Fig. 3.- Velocity profile of an $e^{ \pm}$wind with optical depth $\tau_{0}=3$. 


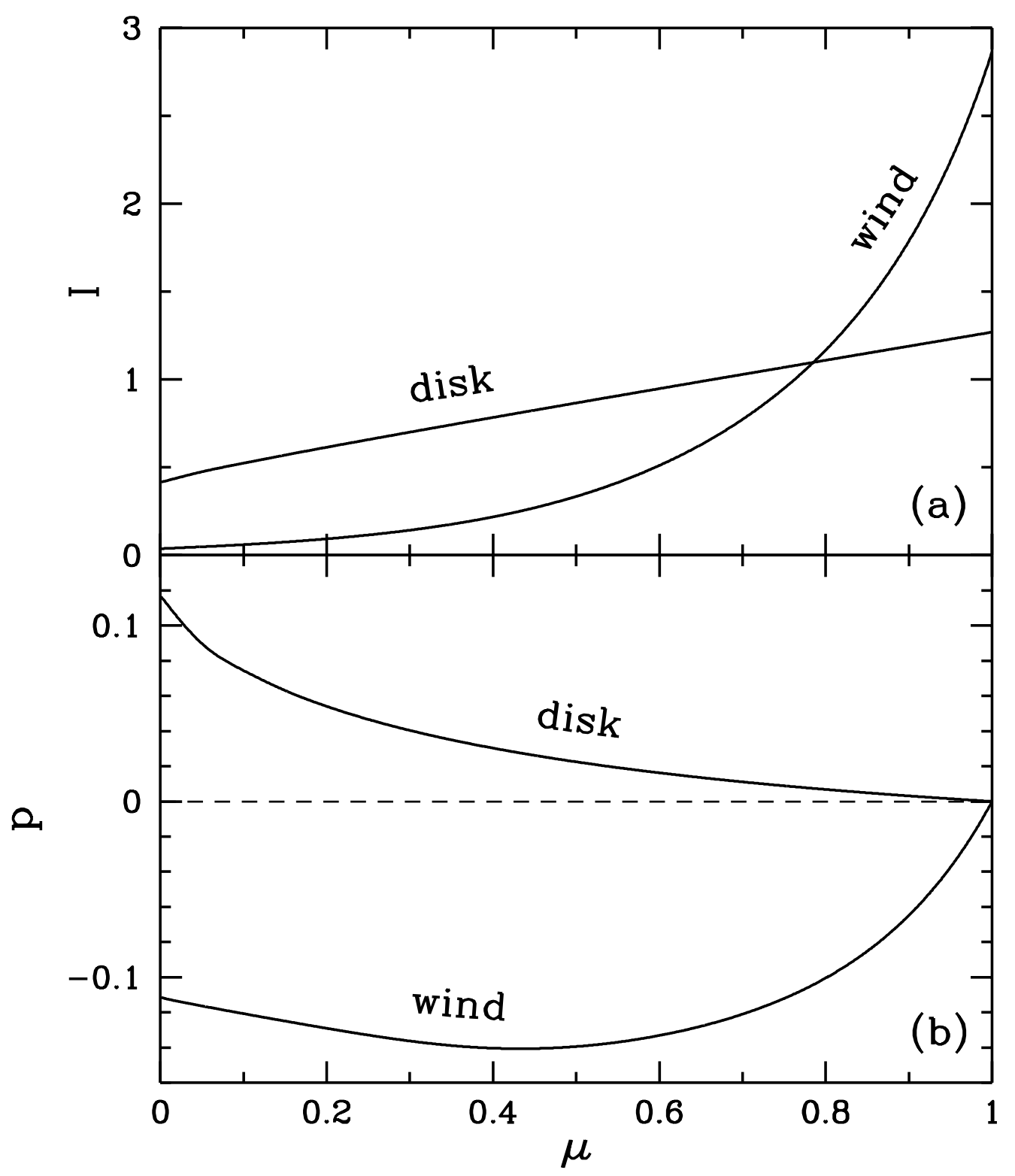

Fig. 4.- Radiation emerging from an optically thick $e^{ \pm}$wind with $\tau_{0}=3$ in comparison to the Chandrasekhar's radiation of the disk: (a) - the angular dependence of the total intensity (normalized so that the net flux equals $\pi$ ), (b) - the angular dependence of the degree of polarization. 